mitigée, sur la base du constat que nombre d'études cliniques ultérieures ne répondent toujours pas aux exigences scientifiques désormais établies, soit parce qu'elles ne les ont pas suivies, soit parce qu'elles omettent de fournir les informations suffisantes. Il faut remarquer également que les autorités d'enregistrement (FDA ou EMA par exemple) ont varié dans leurs exigences.

Les premières études cliniques «coopératives» du siècle dernier ont été menées, aux Etats-Unis, en Grande-Bretagne aussi bien qu'en Suède, dans le cadre d'institutions publiques, l'industrie pharmaceutique fournissant les agents thérapeutiques. L'auteur souligne, dans le chapitre 5, la dérive progressive ayant mené à la prise en charge et au contrôle des essais cliniques par les seules industries pharmaceutiques. Cette situation est d'autant plus paradoxale que, si le développement des médicaments commercialisés par l'industrie a été, dans la plupart des cas, soutenu initialement par des financements publics (de centres universitaires notamment), les bénéfices ultérieurs reviennent aux seules firmes pharmaceutiques. Mais le risque le plus grand tient sans doute au fait que la recherche de profit aboutisse à une perte générale de confiance dans les essais cliniques, avec le soupçon systématique qu'ils ont été orientés et biaisés en fonction des intérêts de la firme ayant commercialisé le produit.

Ces constats décevants, qui ont par ailleurs été présentés et développés dans nombre d'articles et d'ouvrages de cette dernière décennie, justifient, selon la conclusion de l'auteur, la proposition que les essais cliniques soient dans l'avenir administrés par une agence publique indépendante, à même d'en assurer la valeur scientifique et éthique. Mais, comme le dit l'auteur, il s'agit là d'un «enjeu considérable et d'un immense défi».

En conclusion, ce petit ouvrage rappelle utilement les principales étapes, historiques et épistémologiques, de la mise au point des essais cliniques jusqu'au stade actuel de la «médecine des preuves», corrige un certain nombre d'idées reçues à leur sujet, et propose un nouveau cadre de réflexion relatif au statut des essais cliniques de médicaments, au vu des dérives mercantiles qui les ont marqués récemment, où les intérêts du marché et les perspectives de profits ont pris le pas sur les priorités scientifiques et de santé publique.

Jacques Diezi, Lausanne $(\mathrm{CH})$

\title{
Krankenhausgeschichte heute. Was heißt und zu welchem Ende studiert man Hospi- tal- und Krankenhausgeschichte? Herausgegeben von Gunnar Stollberg, Christina Vanja, Ernst Kraas. Berlin, Lit, 2011. 340 S. Ill. (Historia Hospitalium, Bd. 27). € 34.90. ISBN 978-3-643-11287-3
}

$\mathrm{Au}$ moment où dans l'espace francophone certains regrettent la léthargie ${ }^{1}$ dans laquelle est entrée l'histoire des hôpitaux et s'interrogent sur les pistes qu'elle pourrait emprunter, ${ }^{2}$ des réponses convaincantes viennent d'outre-Rhin sous la forme de ce fort volume d'Historia hospitalium qui rappelle qu'il existe en Allemagne depuis quarante ans une société d'histoire des hôpitaux dont l'équivalent français, aux ambitions bien plus modestes, pourrait s'inspirer.

1 A l'exception du récent ouvrage de Chevandier, Christian, L'hôpital dans la France du $X X^{e}$ siècle (Paris, 2009).

2 En particulier dans le cadre du très récent Réseau des historiens universitaires de la médecine (RHUM), voir http://rhum.hypotheses.org/. 
On sait combien, en particulier sous l'impulsion de Axel Heinrich Murken, ${ }^{3}$ l'histoire hospitalière allemande a privilégié les approches architecturales. A lire la deuxième partie de l'ouvrage qui retranscrit les communications prononcées lors du dernier congrès de la société, tenu à Paris en septembre 2010, on mesure combien cette tradition reste forte. En effet, le large thème retenu: Les hôpitaux en France, Allemagne, Pologne (en fait d'anciens territoires allemands): influences et développements (1780-2010) donne une part presque exclusive aux transformations architecturales et à leurs acteurs. Président d'honneur de la société, Axel Murken livre une large synthèse qui va des projets de reconstruction de l'Hôtel-Dieu de Paris à l'hôpital en hauteur, en passant par l'hôpital pavillonnaire (pp. 149-180). Autant l'hôpital Lariboisière est imité en Allemagne comme à Berlin (Friedrichshain et Charlottenbourg), autant l'hôpital en hauteur (Beaujon) ne fait guère école malgré quelques exceptions (Martin-Luther-Krankenhaus à Berlin 1929-1931, Hôpital de SchwäbischHall 1931-1938) et les préconisations des architectes Hermann Distel (1875-1945), Ernst Kropp (1890-1962) et Richard Schachner (1873-1936). Avec l'architecte Peter Pawlik (pp.181-222), on passe de la synthèse à la série de monographies descriptives dans lesquelles se succèdent de splendides gravures, photos et plans accompagnés de brèves légendes. Si la contribution est plus précise que celle de Murken, on y chercherait en vain les explications et les hypothèses qui rendraient compte de ces choix architecturaux différents entre les pays et de leur localisation. Deux autres contributions s'intéressent aux acteurs de ces constructions ou de ces projets. L'une, par Wolfram Otto (pp.239-258), est consacrée à Jacques Tenon (1724-1816), que l'on cite beaucoup mais que l'on connaît peu. Hélas, la biographie esquissée ici n'est ni très précise ni suffisamment contextualisée, l'avalanche de citations de l'intéressé ne pouvant tenir lieu d'analyse. La biographie de Heino Schmieden (1835-1913) associé de Martin Gropius (1824-1880), dressée par Oleg Peters (pp.297-317), est à l'évidence plus originale et plus riche, même si elle reste purement factuelle. Deux autres contributions complètent bien cette vision classique de l'histoire hospitalière puisqu'il s'agit d'études concernant les hôpitaux d'une région. Celle de Bernhard Jungnitz (pp.321-330) consacrée aux établissements hospitaliers du district (Kreis) d'Unna en Rhénanie-du-Nord-Westphalie, se contente d'un recensement et d'une brève description de chacun des nombreux établissements qui couvrent ce petit territoire. Celle que l'historien polonais Piotr Gerber (pp. 259-270) consacre à la modernisation des hôpitaux historiques de Basse-Silésie est plus dense. Elle nous vaut un recueil de splendides photographies des nombreux établissements construits à l'époque de la Silésie allemande (ce que l'article ne dit pas clairement), dont on apprend qu'ils sont toujours en fonction. Par ailleurs, le thème de la reconversion des hôpitaux classés ou historiques abordé ici est un des enjeux de la politique hospitalière contemporaine bien au-delà de cet exemple particulier. Dans celui-ci, il est vraisemblable qu'être hérité de l'époque allemande est, dans une Pologne qui n'avoue guère cet héritage, certainement une difficulté supplémentaire.

A lire cette deuxième partie de l'ouvrage, on pourrait avoir l'impression que l'histoire hospitalière allemande reste fidèle à sa tradition avec ses forces (la précision,

3 Die bauliche Entwicklung des allgemeinen Krankenhauses im 19. Jahrhundert (Göttingen, 1979). Vom Armenhospital zum Grossklinikum: die Geschichte des Krankenhauses vom 18. Jahrhundert bis zur Gegenwart (Köln, 1988, $3^{e}$ éd. 1995). 
l'érudition) et ses faiblesses (la polarisation sur l'architecture, l'oubli partiel de la société). Pourtant, le titre du numéro et les quinze courtes premières contributions qui couvrent 140 pages montrent que les choses ne sont pas aussi simples. Le titre un peu lourd en français «que signifie et dans quel but étudie-t-on l'histoire hospitalière» ou le plus accrocheur «Où vas-tu histoire hospitalière?» choisi par Cornelius Borck pour sa contribution (pp. 17-22) signalent bien la volonté de problématiser cette histoire et de remettre en cause les impensés de l'histoire hospitalière qu'elle soit allemande, française ou d'autres pays. Certes, comme le rappelle Volker Hess (pp. 43-52) dans sa contribution, Alfons Labisch avait dénoncé dès 1980 une histoire hospitalière réduite à celle de l'architecture et de la construction. Certes, et c'est tout à son honneur, Historia hospitalium avait accepté de publier cette charge contre ellemême ${ }^{4}$ mais la citadelle n'en avait pas été ébranlée pour autant. Aujourd'hui, comme nous l'apprend Heiner Fangerau (p. 63-70), c'est une nouvelle équipe, en majorité constituée par des historiens du social et des sociologues comme Gunnar Stollberg 5 qui prend les rênes de la revue et de la société, même si elle ménage l'ancienne équipe, ce qui nous donne l'occasion de lire presque deux livres en un seul. La première partie rappelle que dès les années 1970, et plus en Allemagne qu'ailleurs, on se mit un peu partout à compter et à calculer à partir des registres des patients (Volker Hess). Touchée par le paradigme de l'histoire sociale, l'histoire hospitalière le fut aussi dans les années 1990 par le cultural turn, la social history of medicine from below, les problématiques de la microhistoire, du genre, du corps, de l'histoire de l'enfance (Iris Ritzmann, pp. 87-94). Les archives hospitalières (surtout celles des hôpitaux psychiatriques) furent - et sont toujours - exploitées pour des études de cas et les hôpitaux considérés comme de simples espaces neutres de l'expérience humaine. Aussi intéressantes que soient ces approches, elles ont néanmoins l'inconvénient de mettre entre parenthèses l'histoire des institutions et de leur influence, un peu comme on le faisait dans les années 1960 en étudiant les archives des hôpitaux pour décrire la misère, comme si les établissements en étaient les réceptacles neutres.

Nombre de contributions remettent heureusement l'hôpital au cœur d'une problématique renouvelée. L'un des présupposés les plus ancrés de l'histoire hospitalière est que le grand hôpital technicisé devrait être l'aboutissement inéluctable de l'évolution. Avec les politiques actuelles qui réduisent le nombre de lits ou d'établissements, regroupent les services et surtout développent les soins ambulatoires, le triomphe du grand hôpital n'apparaît plus que comme une simple étape dans la longue chaîne des institutions et lieux de soins (Cornelius Borck, pp. 17-22). Peut-être cette évolution contemporaine pousse-t-elle les historiens à s'intéresser à d'autres hôpitaux. Martin Scheutz et Alfred Stefan Weiss (pp. 23-32) signalent l'importance des hôpitaux privés dans l'Autriche du début du XX $X^{\mathrm{e}}$ siècle, non seulement à Vienne (28,5\% des lits), mais aussi dans de plus petites villes. Ils notent aussi le dynamisme des petits hôpitaux dans la deuxième moitié du XIX ${ }^{\mathrm{e}}$ siècle,${ }^{6}$ dynamisme dû autant aux initiatives des groupes

4 Historia hospitalium, 13, 1980, pp. 217-233.

$5 \mathrm{Au}$ milieu d'une œuvre considérable, on ne citera que le livre écrit avec Ingo Tamm, Die Binnendifferenzierung in deutschen Krankenhäusern bis zum ersten Weltkrieg (Stuttgart, 2001).

6 Constatable aussi en France Olivier Faure, Splendeur et misère des petits hôpitaux en France au XIX $X^{e}$ et $X X^{e}$ siècles, dans Yannick Marec (éd.), Accueillir ou soigner? L'hôpital et ses alternatives du Moyen-Age à nos jours (Rouen/Le Havre, 2007), 153-166. 
religieux, des associations et des communes qu'aux décisions des autorités médicales et étatiques. De son côté, Cristina Vanja (pp. 95-104) plaide pour élargir l'histoire hospitalière à tous les établissements de santé (Kur- und Heilanstalten difficilement traduisibles par «établissements de cure») qui représentent dans l'Allemagne des années 1920 la moitié des lits d'hospitalisation. Au-delà de leur très grande diversité, tant par les populations accueillies (tuberculeux, nerveux, anémiés, convalescents, etc.) que par les méthodes utilisées (cure d'air, de soleil, d'eau'), ils ont tous en commun de rester fidèles à la diététique entendue comme une façon de vivre près de la nature. Pourtant, loin de représenter une sorte d'antihospitalisation, ils entretiennent des influences réciproques avec les hôpitaux classiques. Ils leur empruntent souvent des équipements techniques de diagnostic les plus modernes et leur inspirent des innovations sur le plan de l'accueil, de l'aération, de l'architecture (jardin, balcons). Ainsi liés, les uns comme les autres révéleraient, en même temps qu'ils la hâteraient, notre acheminement vers une société de santé (Gesundheitsgesellschaft) dans laquelle nous vivons. On pourrait dire la même chose des cliniques ambulatoires qu'étudie Karen Nolte (pp.79-86). Elargie aux autres institutions de santé,l'histoire hospitalière ne saurait s'y réduire. Contrairement à une légende tenace, les hôpitaux (en tant qu'institution) ne se sont jamais occupés que de malades. Annonçant un ouvrage très prochain, le très novateur et productif Michael Stolberg (pp. 115-124) nous offre un premier coup d'œil sur un continent presque ignoré, celui des hospices d'incurables. Il note que les premiers d'entre eux sont apparus en Italie à la fin du $\mathrm{XV}^{\mathrm{e}}$ siècle, donc bien avant la fameuse médicalisation de l'hôpital supposée responsable de leur expulsion, et que dès 1744 à Dublin, les médecins interviennent dans ces asiles pour dispenser des soins qualifiés de palliatifs (palliated). Finalement, la médicalisation, à quoi s'est si souvent résumée l'histoire hospitalière, n'a guère de place et le mot n'apparaît même pas. Ceux qui, comme Irmtraut Sahmland à propos des hôpitaux de Hesse (pp. 53-62), font allusion à ce processus insistent tant sur la subtilité et la complexité d'un processus sur lequel on est finalement si peu renseigné concrètement que l'on finit par douter de son importance, sinon de son existence.

Une dernière série de contributions invite à intégrer l'histoire hospitalière dans l'histoire générale. Fritz Dross (pp.33-42) signale à juste titre qu'à l'époque moderne comme vers 1900, les hôpitaux sont des acteurs économiques et urbains essentiels qui jouent un rôle essentiel dans l'autoreprésentation des villes. Faut-il aller jusqu'à dire comme l'auteur que «l'histoire de l'hôpital c'est l'histoire de la ville»? Oui sans doute, à condition de ne pas dissoudre la première dans la seconde. Ernest Kraas et Anne-Marie Frybourg affirment que, si à première vue l'histoire de l'hôpital est très étroite et très spécialisée, elle est en fait le lieu de convergences de plusieurs disciplines. Plus encore, avec ces deux auteurs, on peut estimer que cette institution dense et multiple mérite des approches de même nature comme celles de ce très riche et stimulant numéro. On peut souhaiter que le rapprochement entre les deux traditions d'histoire hospitalière amorcé ici se renforce et que le renouveau de l'histoire hospitalière suggéré ici fasse école en particulier dans l'espace francophone.

Olivier Faure, Lyon (F)

7 Voir à ce titre Wolff, Eberhard (éd.), Lebendige Kraft: Max Bircher-Benner und sein Sanatorium im historischen Kontext (Baden, 2010). 